\title{
BIBLIOGRAFÍA ESPECIALIZADA SOBRE TRADUCCIÓN E INTERPRETACIÓN: LA COLECCIÓN TRADUCCIÓN EN EL ATRIO DE LA EDITORIAL ATRIO (2003-2012) \\ Emilio Ortega Arjonilla \\ Universidad de Málaga
}

\section{ABSTRACT}

This bibliographic article collects the books published in the Collection Traducción en el Atrio, by the publishing house Editorial Atrio (Granada, Spain). We thus present all the works published between 2003 and 2012, divided into different sections according to different domains of the Translation Studies and related disciplines (Translation Theory, Conference Interpreting, Social Interpreting, Terminology, Applied Linguistics and Translation, etc.).

KEYWORDS: Bibliography, Translation theory, Conference Interpreting, Social Interpreting, Terminology, Applied Linguistics and Translation

\section{RESUMEN}

El presente artículo bibliográfico recoge las obras publicadas en la colección Traducción en el Atrio de la Editorial Atrio (Granada, España). Presentamos todas las obras publicadas en esta editorial entre 2003 y 2012 dentro de esta colección, divididas en distintas secciones relacionadas con ámbitos de los Estudios de Traducción e Interpretación y disciplinas relacionadas (Teoría de la traducción, Interpretación de conferencias, Interpretación Social, Terminología, Lingüística Aplicada y Traducción, etc.).

PALABRAS CLAVE: Bibliografía, Teoría de la traducción, Interpretación de Conferencias, Interpretación social, Terminología, Lingüística Aplicada y Traducción

\section{INTRODUCCIÓN}

La colección Traducción en el atrio de la Editorial Atrio de Granada es una de las colecciones de referencia, a escala nacional e internacional, en la difusión 
ENTRECULTURAS Número 5. ISSN: 1989-5097. Fecha de publicación: 23-01-2013

de resultados de investigación dentro del área de conocimiento de traducción e interpretación, sobre todo en la difusión de la literatura traductológica en español. Aparece indexada en el SPI Scholarly Publishers Indicators for Social Sciences and Humanities, donde se le concedieron 1,32 puntos, muy por delante de algunas editoriales españolas comerciales y de casi todas las editoriales universitarias del Estado, de un total de más de 200 editoriales nacionales evaluadas.

Entre las bases de datos nacionales e internacionales en las que aparecen recogidos títulos de esta colección podemos citar, entre otras, las siguientes: The Library of Congress (Washington, EEUU), The Library of Congress Authorities (Washington, EEUU), The Library of Congress Online Catalog (Washington, EEUU), la Biblioteca de Traducción del Colegio de Traductores Públicos de Buenos Aires (Argentina), la BNF (Bibliothèque Nationale de France, París), Les repertoires spécialisés de l'ISIT (Université Catholique de París, Francia), Le Fonds Documentaire-Langage del ISIT de París (Université Catholique de París, Francia) y de la ETI (Genève, Suiza), The University of Toronto Libraries, WORLDCAT, WORLDCAT IDENTITIES, Translation Journal Abstracts (Ed. St. Jerome, Reino Unido), etc. También aparece indexada, a escala nacional, en las bases de datos BITRA (Universidad de Alicante), Biblioteca Virtual Cervantes (Instituto Cervantes) y DIALNET (Universidad de La Rioja).

Esta colección cuenta, entre 2003 y 2012, con 24 títulos publicados, entre monografías individuales y colectivas, algunas con varias ediciones $\mathrm{O}$ reimpresiones en su haber.

En cuanto a las temáticas abordadas en los títulos publicados en la Editorial Atrio, podemos destacar, por orden de importancia cuantitativa, los siguientes:

\begin{tabular}{|l|l|}
\hline $\begin{array}{l}\text { Ámbito o ámbitos a los que se dedica la } \\
\text { monografía }\end{array}$ & $\begin{array}{l}\text { Número de títulos publicados } \\
\text { dentro de este ámbito o ámbitos }\end{array}$ \\
\hline $\begin{array}{l}\text { 1. Estudios transversales o interdisciplinares } \\
\text { relacionados con la traducción (perspectivas } \\
\text { teóricas, didácticas o profesionales) }\end{array}$ & 5 títulos publicados \\
\hline $\begin{array}{l}\text { 2. Teoría de la traducción e investigación } \\
\text { traductológica }\end{array}$ & $\begin{array}{l}6 \text { títulos publicados (aunque } 4 \text { de } \\
\text { ellos corresponden a ediciones } \\
\text { sucesivas de la monografía no 1) }\end{array}$ \\
\hline $\begin{array}{l}\text { 3. Terminología y lexicografía, terminología } \\
\text { y traducción, historia de la terminología }\end{array}$ & 4 títulos publicados \\
\hline $\begin{array}{l}\text { 4. Teoría, didáctica y práctica de la } \\
\text { interpretación de conferencias (incluyendo } \\
\text { los que también abordan la traducción) }\end{array}$ & 4 títulos publicados \\
\hline
\end{tabular}


ENTRECULTURAS Número 5. ISSN: 1989-5097. Fecha de publicación: 23-01-2013

\begin{tabular}{|l|l|l|}
\hline $\begin{array}{l}\text { 5. Didáctica de la traducción (general o } \\
\text { especializada), didáctica de la L2 }\end{array}$ & 3 títulos publicados \\
\hline $\begin{array}{l}\text { 6. Historia de la traducción y de la } \\
\text { traductología }\end{array}$ & 2 títulos publicados \\
\hline $\begin{array}{l}\text { 7. Traducción especializada, TIC y } \\
\text { traducción }\end{array}$ & 2 títulos publicados \\
\hline $\begin{array}{l}\text { 8. Mundo profesional de la traducción e } \\
\text { interpretación }\end{array}$ & 1 título publicado \\
\hline
\end{tabular}

Los títulos que corresponden a cada uno de los ámbitos enunciados más arriba son los siguientes:

1.1. TÍtUllos SOBRE ESTUDIOS TRANSVERSALES O INTERDISCIPLINARES RELACIONADOS CON LA TRADUCCIÓN (PERSPECTIVAS TEÓRICAS, DIDÁCTICAS O PROFESIONALES)

F. J. GARCÍA MARCos, M. Á. GARCíA PEINADO, E. ORTEGA ARJONILLA y N. PERDU HONEYMAN (eds.): Traducción, cultura $e$ inmigración. Reflexiones interdisciplinares. Editorial Atrio, colección Traducción en el atrio $n^{\circ}$ 6, Granada: 2004. ISBN: 978-84-96101-25-8.

Nicolás A. CAMPOS PLAZA, Miguel A. GARCÍA PEINADO, Emilio ORTEGA ARJONILLA y Cécile VILVANDRE DE SOUSA (eds.): El español, lengua de cultura, lengua de traducción. Aspectos teóricos, metodológicos y profesionales. Ediciones de la Universidad de Castilla-La Mancha / Editorial Atrio, colección Traducción en el atrio no 7, Granada: 2005. ISBN: 978-8496101-28-9.

Nicolás A. CAMPOS PLAZA y Emilio ORTEGA ARJONILLA: Panorama de Lingüistica y Traductología. Ediciones de la Universidad de Castilla-La Mancha / Editorial Atrio, colección Traducción en el atrio no 8, Granada: 2005. ISBN: 978-84-96101-38-8.

María del Carmen BALBUENA TOREZANO y Ángeles GARCÍA CALDERÓN (eds.): Traducción y mediación cultural. Reflexiones interdisciplinares. Editorial Atrio, colección Traducción en el atrio n 12, Granada: 2006. ISBN: 978-84-96101-54-8.

Emilio ORTEGA ARJONILLA y Maria Joao MARÇALO (eds.): Linguistica e Traducao na Sociedade do Conhecimento. Universidade de Évora (col. Lingüística 6) / Editorial Atrio, colección Traducción en el atrio n ${ }^{\circ} 21$, 2010. ISBN (Atrio, Granada): 978-84-96101-93-7. ISBN (Évora, Linguística): 978-972-99292-3-6. 
1.2. Títulos SOBRE TEORÍA DE LA TRADUCCIÓN E INVESTIGACIÓN TRADUCTOLÓGICA, (ORDENADOS POR FECHA DE PUBLICACIÓN)

Emilio ORTEGA ARJONILLA (dir.): Panorama actual de la investigación en traducción e interpretación (vol. 1). Editorial Atrio, colección Traducción en el atrio $\mathrm{n}^{\mathrm{o}}$ 1, Granada: 2003 (1 ${ }^{\mathrm{a}}$ ed. en 2 vols.). ISBN (vol. 1): 84-96101-10-X. ISBN (obra completa): 84-96101-12-6.

Miguel Ángel GARCÍA PEINADO (dirs.): Panorama actual de la investigación en traducción e interpretación (vol. 1). Editorial Atrio, colección Traducción en el atrio no 1, Granada: 2003 (1 ${ }^{\text {a }}$ ed. en 2 vols.). ISBN (vol. 2): 84-96101-11-X. ISBN (obra completa): 84-96101-12-6.

Emilio ORTEGA ARJONILLA (dir.): Panorama actual de la investigación en traducción e interpretación (3 vols.). Editorial Atrio, colección Traducción en el atrio $n^{\circ} 1$, Granada: 2004 ( $2^{\text {a }}$ ed., corregida y aumentada, en 3 vols.). ISBN: 84-96101-26-6.

Emilio ORTEGA ARJONILLA, Ana Belén MARTÍNEZ LÓPEZ y Elena ECHEVERRÍA PEREDA (eds.): Panorama actual de la investigación en traducción e interpretación (3 vols.). Editorial Atrio, colección Traducción en el atrio $\mathrm{n}^{\mathrm{o}}$ 1, Granada: 2003 ( $1^{\mathrm{a}}$ ed. en 2 vols.), 2004 ( $2^{\mathrm{a}}$ ed., corregida y aumentada en 3 vols.), 2010 ( $3^{\text {a }}$ ed. corregida y aumentada en 3 vols.). ISBN: 978-84-96101-78-4.

Emilio ORTEGA ARJONILLA, Juan Pedro MONFERRER-SALA y Vicente LÓPEZ FOLGADO (eds.): Eugene A. Nida, pionero de la traductología contemporánea. Universidad de Córdoba-Editorial Atrio, colección Traducción en el atrio no 11, Granada: 2006. ISBN: 978-84-96101-52-4.

Emilio ORTEGA ARJONILLA, Leandro FÉLIX FERNÁNDEZ, Ana Belén MARTÍNEZ LÓPEZ e Iván DELGADO PUGÉS: El papel del contexto en traducción e interpretación. Editorial Atrio, colección Traducción en el atrio $\mathrm{n}^{\mathrm{o}}$ 16, Granada: 2009. ISBN: 978-84-96101-75-3.

1.3. Títulos SOBRE TERMinOlogíA Y LEXICOGRAFÍA, TERMINOLOGÍA Y TRADUCCIÓN, HISTORIA DE LA TERMINOLOGÍA (ORDENADOS POR FECHA DE PUBLICACIÓN)

Natividad GALLARDO SAN SALVADOR (dir.): Terminología y traducción: un bosquejo de su evolución. Editorial Atrio, colección Traducción en el atrio n ${ }^{\circ} 2$, Granada: 2003. ISBN: 978-84-96101-16-6.

Natividad GALLARDO SAN SALVADOR, y J. GÓMEZ DE ENTERRÍA (eds.): Estudios de lexico especializado: Economía actual (1998-2008). Migraciones, 
gestión del patrimonio cultural y gestion del turismo gastronómico. Editorial Atrio, colección Traducción en el atrio no 15, Granada: 2009. ISBN: 978-8496101-73-9.

Bernard THIRY: Terminología y derecho. La responsabilidad civil extracontractual. Contribución a su tratamiento terminográfico y a la Teoría de la Terminología. Editorial Atrio, colección Traducción en el atrio n ${ }^{0}$ 18, Granada: 2009. ISBN: 978-84-96101-79-1.

Galyna G. VERBA y Rafael GUZMÁN TIRADO: Guía de conversación, ucraniano. Editorial Atrio, colección Traducción en el atrio no 20, Granada: 2010. ISBN: 978-84-96101-17-3.

1.4. TÍTUlOS SOBRE TEORÍA, DIDÁCTICA Y PRÁCTICA DE LA INTERPRETACIÓN DE (INCLUYENDO LOS QUE TAMBIÉN ABORDAN LA TRADUCCIÓN)

Dorothy KELLY, Anne MARTIN, Marie-Louise NOBS, Dolores SÁNCHEZ y Catherine WAY (eds.): La direccionalidad en traducción e interpretación: perspectivas teóricas, profesionales y didácticas. Editorial Atrio, colección Traducción en el atrio no 5, Granada: 2003. ISBN: 84-96101-09-6.

Jesús DE MANUEL JEREZ (coord.): Nuevas tecnologías y formación de intérpretes. Editorial Atrio, colección Traducción en el atrio no 4, Granada: 2003. ISBN: 978-84-96101-14-2.

Presentación PADILLA BENÍTEZ, Pedro MACIZO SORIA y María Teresa BAJO MOLINA: Tareas de traducción e interpretación desde una perspectiva cognitiva. Una propuesta integradora. Editorial Atrio, colección Traducción en el atrio no 13, Granada: 2007. ISBN: 978-84-96101-58-6.

Luis ALONSO BACIGALUPE: El procesamiento de la información durante la interpretación simultánea: un modelo en tres niveles. Editorial Atrio, colección Traducción en el atrio no 19, Granada: 2009. ISBN: 978-84-96101-65-4

1.5. Títulos SOBRE DIDÁCTICA DE LA TRADUCCIÓN (GENERAL O ESPECIALIZADA) Y DIDÁCTICA DE LA L2

David ATKINSON, Marián MORÓN y Dorothy KELLY (eds.): Teaching in the multicultural classroom at the University: the Temcu project. Editorial Comares, colección Traducción en el atrio no 10, Granada: 2006. ISBN: 978-8496101-41-8.

Esperanza ALARCÓN NAVÍO (ed.): La traducción en contextos especializados. Propuestas didácticas. Editorial Atrio, colección Traducción en el atrio $\mathrm{n}^{\mathrm{o}} 22$, 2010. ISBN: 978-84-96101-86-9. 
Óscar DÍAZ FOUCES (ed.): Olhares \& Miradas. Reflexiones sobre la traducción portugués-español y su didáctica. Editorial Atrio, colección Traducción en el atrio no 24, Granada: 2012. ISBN: 978-84-15275-10-7.

\subsection{TÍTULOS SOBRE HISTORIA DE LA TRADUCCIÓN Y DE LA TRADUCTOLOGÍA}

Juan Jesús ZARO (ed.): Diez estudios sobre la traducción en la España del siglo XIX. Editorial Atrio, colección Traducción en el atrio no 14, Granada: 2008. ISBN: 978-84-96101-63-0

Carmen ACUÑA PARTAL y Marcos RODRÍGUEZ ESPINOSA: Archivo y edición digital de textos literarios y ensayísticos traducidos al español y tratados sobre traducción del siglo XIX. Editorial Atrio, colección Traducción en el atrio $\mathrm{n}^{\mathrm{o}}$ 17, Granada: 2009. ISBN: 978-84-96101-64-7.

\subsection{TÍTULOS SOBRE TRADUCCIÓN ESPECIALIZADA, TIC Y TRADUCCIÓN}

Gloria CORPAS PASTOR y María José VARELA SALINAS (eds.): Entornos informáticos de la traducción profesional: las memorias de traducción. Editorial Atrio, colección Traducción en el atrio no 3, Granada: 2003. ISBN: 978-8496101-15-9.

Adrián FUENTE LUQUE (ed.): La Traducción en el sector turístico. Editorial Atrio, colección Traducción en el atrio no 9, Granada: 2005. ISBN: 8496101-40-1.

1.8. TÍtulos SOBRE EL MUNDO PROFESIONAL DE LA TRADUCCIÓN E INTERPRETACIÓN

VVAA (eds.): Inserción profesional d@s estudiantes de traducción e interpretación. Editorial Atrio, colección Traducción en el atrio no 23, Granada: 2011. ISBN: 978-84-96101-90-6.

2. LA COLECCIÓN TRADUCCIÓN EN EL ATRIO (2003-2012) NÚMERO A NÚMERO (MONOGRAFÍAS ORDENADAS POR FECHA DE PUBLICACIÓN)

Los 24 títulos que componen esta colección, ordenados por fecha de publicación, son los siguientes: 
$\mathbf{N}^{\circ}$ 1. Emilio ORTEGA ARJONILLA (dir.): Panorama actual de la investigación en traducción e interpretación (vol. 1). Editorial Atrio, colección Traducción en el atrio $n^{\circ}$ 1, Granada: 2003 ( $1^{a}$ ed. en 2 vols.). ISBN (vol. 1): 8496101-10-X. ISBN (obra completa): 84-96101-12-6.

$\mathbf{N}^{\mathbf{0}}$ 1. Miguel Ángel GARCÍA PEINADO (dirs.): Panorama actual de la investigación en traducción e interpretación (vol. 1). Editorial Atrio, colección Traducción en el atrio $n^{\circ}$ 1, Granada: 2003 ( $1^{a}$ ed. en 2 vols.). ISBN (vol. 2): $84-$ 96101-11-X. ISBN (obra completa): 84-96101-12-6.

$\mathbf{N}^{\mathbf{0}}$ 2. Natividad GALLARDO SAN SALVADOR (dir.): Terminología $y$ traducción: un bosquejo de su evolución. Editorial Atrio, colección Traducción en el atrio no 2, Granada: 2003. ISBN: 978-84-96101-16-6.

$\mathbf{N}^{\circ}$ 3. Gloria CORPAS PASTOR y María José VARELA SALINAS (eds.): Entornos informáticos de la traducción profesional: las memorias de traducción. Editorial Atrio, colección Traducción en el atrio no 3, Granada: 2003. ISBN: 978-8496101-15-9.

$\mathbf{N}^{\circ}$ 4. Jesús DE MANUEL JEREZ (coord.): Nuevas tecnologias y formación de intérpretes. Editorial Atrio, colección Traducción en el atrio no 4, Granada: 2003. ISBN: 978-84-96101-14-2.

$\mathbf{N}^{\mathbf{0}}$ 5. Dorothy KELLY, Anne MARTIN, Marie-Louise NOBS, Dolores SÁNCHEZ y Catherine WAY (eds.): La direccionalidad en traducción e interpretación: perspectivas teóricas, profesionales y didácticas. Editorial Atrio, colección Traducción en el atrio no 5, Granada: 2003. ISBN: 84-96101-09-6.

No 6. F. J. GARCÍA MARCOS, M. Á. GARCÍA PEINADO, E. ORTEGA ARJONILLA y N. PERDU HONEYMAN (eds.): Traducción, cultura e inmigración. Reflexiones interdisciplinares. Editorial Atrio, colección Traducción en el atrio no 6, Granada: 2004. ISBN: 978-84-96101-25-8.

- $\mathbf{N}^{\mathbf{0}}$ 1. Emilio ORTEGA ARJONILlA (dir.): Panorama actual de la investigación en traducción e interpretación (3 vols.). Editorial Atrio, colección Traducción en el atrio $n^{\circ} 1$, Granada: 2004 ( $2^{a}$ ed., corregida y aumentada, en 3 vols.). ISBN: 84-96101-26-6.

$\mathbf{N}^{\circ}$ 7. Nicolás A. CAMPOS PLAZA, Miguel A. GARCÍA PEINADO, Emilio ORTEGA ARJONILLA y Cécile VILVANDRE DE SOUSA (eds.): El español, lengua de cultura, lengua de traducción. Aspectos teóricos, metodológicos y profesionales. Ediciones de la Universidad de Castilla-La Mancha / Editorial Atrio, colección Traducción en el atrio no 7, Granada: 2005. ISBN: 978-8496101-28-9.

No 8. Nicolás A. CAMPOS PLAZA y Emilio ORTEGA ARJONILLA: Panorama de Lingüistica y Traductología. Ediciones de la Universidad de Castilla-La Mancha / Editorial Atrio, colección Traducción en el atrio $n^{\circ}$ 8, Granada: 2005. ISBN: 978-84-96101-38-8. 
$\mathbf{N}^{\mathbf{0}}$ 9. Adrián FUENTE LUQUE (ed.): La Traducción en el sector turístico. Editorial Atrio, colección Traducción en el atrio no 9, Granada: 2005. ISBN: 84-96101-40-1.

No 10. David ATKINSON, Marián MORÓN y Dorothy KELLY (eds.): Teaching in the multicultural classroom at the University: the Temcu project. Editorial Comares, colección Traducción en el atrio n 10, Granada: 2006. ISBN: 97884-96101-41-8.

No 11. Emilio ORTEGA ARJONILLA, Juan Pedro MONFERRER-SALA y Vicente LÓPEZ FOLGADO (eds.): Eugene A. Nida, pionero de la traductología contemporánea. Universidad de Córdoba-Editorial Atrio, colección Traducción en el atrio no 11, Granada: 2006. ISBN: 978-84-96101-52-4.

No 12. María del Carmen BALBUENA TOREZANO y Ángeles GARCÍA CALDERÓN (eds.): Traducción y mediación cultural. Reflexiones interdisciplinares. Editorial Atrio, colección Traducción en el atrio n ${ }^{\circ}$ 12, Granada: 2006. ISBN: 978-84-96101-54-8.

No 13. Presentación PADILLA BENÍTEZ, Pedro MACIZO SORIA y María Teresa BAJO MOLINA: Tareas de traducción e interpretación desde una perspectiva cognitiva. Una propuesta integradora. Editorial Atrio, colección Traducción en el atrio no 13, Granada: 2007. ISBN: 978-84-96101-58-6.

$\mathbf{N}^{\mathbf{0}}$ 14. Juan Jesús ZARO (ed.): Diez estudios sobre la traducción en la España del siglo XIX. Editorial Atrio, colección Traducción en el atrio n ${ }^{\circ} 14$, Granada: 2008. ISBN: 978-84-96101-63-0

No 15. Natividad GALLARDO SAN SALVADOR, y J. GÓMEZ DE ENTERRÍA (eds.): Estudios de lexico especializado: Economía actual (1998-2008). Migraciones, gestión del patrimonio cultural y gestion del turismo gastronómico. Editorial Atrio, colección Traducción en el atrio n ${ }^{\circ}$ 15, Granada: 2009. ISBN: 978-8496101-73-9.

No 16. Emilio ORTEGA ARJONILLA, Leandro FÉLIX FERNÁNDEZ, Ana Belén MARTÍNEZ LÓPEZ e Iván DELGADO PUGÉS: El papel del contexto en traducción e interpretación. Editorial Atrio, colección Traducción en el atrio no 16, Granada: 2009. ISBN: 978-84-96101-75-3.

No 17. Carmen ACUÑA PARTAL y Marcos RODRÍGUEZ ESPINOSA: Archivo y edición digital de textos literarios $y$ ensayisticos traducidos al español y tratados sobre traducción del siglo XIX. Editorial Atrio, colección Traducción en el atrio $\mathrm{n}^{\circ}$ 17, Granada: 2009. ISBN: 978-84-96101-64-7.

No 18. Bernard THIRY: Terminología y derecho. La responsabilidad civil extracontractual. Contribución a su tratamiento terminográfico y a la Teoría de la Terminología. Editorial Atrio, colección Traducción en el atrio n ${ }^{\circ} 18$, Granada: 2009. ISBN: 978-84-96101-79-1. 
$\mathbf{N}^{\mathbf{0}}$ 19. Luis ALONSO BACIGALUPE: El procesamiento de la información durante la interpretación simultánea: un modelo en tres niveles. Editorial Atrio, colección Traducción en el atrio no 19, Granada: 2009. ISBN: 978-84-96101-65-4

- $\mathbf{N}^{\mathbf{0}}$ 1. Emilio ORTEGA ARJONILLA, Ana Belén MARTÍNEZ LÓPEZ y Elena ECHEVERRÍA PEREDA (eds.): Panorama actual de la investigación en traducción e interpretación (3 vols.). Editorial Atrio, colección Traducción en el atrio n ${ }^{\mathrm{o}}$ 1, Granada: 2010 ( $3^{\mathrm{a}}$ ed. corregida y aumentada en 3 vols.). ISBN: 978-84-96101-78-4.

No 20. Galyna G. VERBA y Rafael GUZMÁN TIRADO: Guía de conversación, ucraniano. Editorial Atrio, colección Traducción en el atrio $\mathrm{n}^{\circ} 20$, Granada: 2010. ISBN: 978-84-96101-17-3.

No 21. Emilio ORTEGA ARJONILLA y Maria Joao MARÇALO (eds.): Linguistica e Traduça na Sociedade do Conbecimento. Universidade de Évora (col. Lingüística 6) / Editorial Atrio, colección Traducción en el atrio n ${ }^{\circ}$ 21, 2010. ISBN (Atrio, Granada): 978-84-96101-93-7. ISBN (Évora, Linguística): 978972-99292-3-6.

No 22. Esperanza ALARCÓN NAVÍO (ed.): La traducción en contextos especializados. Propuestas didácticas. Editorial Atrio, colección Traducción en el atrio $n^{\circ} 22,2010$. ISBN: 978-84-96101-86-9.

$\mathbf{N}^{\mathbf{0}}$ 23. Luis ALONSO BACIGALUPE (eds.): Inserción profesional d@s estudiantes de traducción e interpretación. Editorial Atrio, colección Traducción en el atrio no 23, Granada: 2011. ISBN: 978-84-96101-90-6.

No 24. Óscar DÍAZ FOUCES (ed.): Olhares \& Miradas. Reflexiones sobre la traducción portugués-español y su didáctica. Editorial Atrio, colección Traducción en el atrio no 24, Granada: 2012. ISBN: 978-84-15275-10-7.

3. LA REVISTA PUENTES HACLA NUEV AS INVESTIGACIONES EN LA MEDIACIÓN INTERCULTURAL (2002-2010) NÚMERO A NÚMERO

La revista Puentes constituye un proyecto editorial, dirigido por varios profesores del Departamento de Traducción e Interpretación de la Universidad de Granada, con 9 números publicados entre 2002 y 2010 en las editoriales Comares ( $\mathrm{n}^{\circ} 1$ y $\mathrm{n}^{\circ} 2$ ) y Atrio ( $\mathrm{n}^{\circ} 3, \mathrm{n}^{\circ} 4, \mathrm{n}^{\circ} 5, \mathrm{n}^{\circ} 6, \mathrm{n}^{\circ} 7, \mathrm{n}^{\circ} 8$ y $\left.\mathrm{n}^{\circ} 9\right)$ de Granada.

La directora de la revista es la Dra. Presentación Padilla Benítez, catedrática de Traducción e Interpretación de la Universidad de Granada.

Su comité editor está compuesto por los siguientes profesores del Departamento de Traducción e Interpretación de la Universidad de Granada: 
Isabel Abril Martí (Universidad de Granada, FTI)

Dorothy A. Kelly (Universidad de Granada, FTI)

Anne Martin (Universidad de Granada, FTI)

Roberto Mayoral Asensio (Universidad de Granada, FTI)

Catherine Way (Universidad de Granada, FTI)

En cuanto al comité asesor de esta revista, este cuenta con los siguientes expertos nacionales e internacionales:

Raquel de Pedro Álvarez.- Heriot-Watt University, Edimburgo

África Vidal Claramonte.- Universidad de Salamanca

Jesús Baigorri Jalón.- Universidad de Salamanca

Juan Crespo Hidalgo.- Universidad de Málaga

Jorge Diaz-Cintas.- Imperial College, Londres

Ada Franzoni Moldavsky.- Colegio de Traductores Públicos de la

Provincia de Buenos Aires

Natividad Gallardo San Salvador.- Universidad de Granada

Miguel Hagerty Fox.- Universidad de Granada

Amparo Hurtado Albir.- Universidad Autónoma de Barcelona

Óscar Jiménez Serrano.- Universidad de Granada

Christiane Nord.- Fachhochschule Magdeburg

Amparo Jiménez Ivars.- Universitat Jaume I, Castellón de la Plana

Franz Pöchhaker.- Universität Wien, Austria

Bill Richardson.- University of Galway, Irlanda

Silvia Roiss.- Universidad de Salamanca

Louis Jolicoeur.- Université Laval, Quebec

Isabel García Izquierdo.- Universitat Jaume I

Mariachiara Russo.- Università di Bologna

\subsection{LA REVISTA PUENTES (2002-2010) NÚMERO A NÚMERO}

Algo que ha caracterizado a esta revista desde su lanzamiento en enero de 2002 es la alternancia entre números generales y números monográficos dedicados a un ámbito específico de la traducción o la interpretación.

Los números generales publicados son los siguientes:

Puentes $\mathbf{n}^{\mathbf{0}}$ 1. (enero de 2002). Editoras: Presentación Padilla, Dorothy A. Kelly y Anne Martin. 
Puentes $\mathbf{n}^{\mathbf{o}} 3$ (enero de 2004). Editoras: Presentación Padilla, Dorothy A. Kelly y Anne Martin.

Puentes $\mathbf{n}^{\mathbf{0}} \mathbf{5}$ (noviembre de 2005). Editoras: Presentación Padilla y Dorothy A. Kelly.

Puentes $\mathbf{n}^{\mathbf{0}} 7$ (octubre de 2007). Editores: Presentación Padilla, Anne Martin, Roberto Mayoral y Dorothy Kelly.

Puentes $\mathbf{n}^{\mathbf{0}} 9$ (2010). Editores: Marie-Louise Nobs y Jesús de Manuel Jerez.

Los números monográficos publicados son los siguientes:

Puentes $\mathbf{n}^{\mathbf{0}} 2$ (noviembre de 2002). Editora: Catherine Way.

Número monográfico dedicado a la traducción jurídica.

Puentes $\mathbf{n}^{\mathbf{0}} 4$ (octubre de 2004). Editoras: Anne Martin e Isabel Abril Martí.

Número monográfico dedicado a la traducción e interpretación social.

Puentes $\mathbf{n}^{\mathbf{0}} \mathbf{6}$ (noviembre de 2005). Editor: Roberto Mayoral Asensio.

Número monográfico dedicado a la traducción audiovisual.

Puentes $\mathbf{n}^{\mathbf{0}} 8$ (diciembre de 2007). Editor: Manuel C. Feria García.

Número monográfico dedicado a la traducción y la interpretación árabeespañol-árabe.

Todos los números publicados entre 2002 y 2010 de la revista Puentes se encuentran disponibles, en formato PDF, en la siguiente dirección electrónica: www.ugr.es/ greti/revista_puente.htm 